\title{
HGF alleviates septic endothelial injury by inhibiting pyroptosis via the mTOR signalling pathway
}

Fei Peng, Wei Chang, Qin Sun, Xinyi Xu, Jianfeng Xie, Haibo Qiu and Yi Yang ${ }^{*}$ (D)

\begin{abstract}
Background: Endothelial injury is one of the predominant pathophysiological characteristics of sepsis and is the major cause of sepsis-induced multiple organ failure. Endothelial pyroptosis is a fatal mechanism of endothelial injury in sepsis, and specific, effective therapies are lacking. Although hepatocyte growth factor (HGF) has been shown to have anti-apoptotic and anti-necrotic effects, whether it prevents pyroptosis to improve endothelial injury in sepsis remains unclear.

Methods: Recombinant HGF was intravenously injected into mice with sepsis caused by caecal ligation puncture (CLP). Histopathological examination and transmission electron microscopy (TEM) were used to measure lung vascular endothelial injury. Lipopolysaccharide (LPS) was transfected into EA.hy926 cells to induce endothelial pyroptosis, and the cells were treated with HGF in the presence of inhibitors of c-Met and mTOR, namely, PHA665752 and rapamycin, respectively. The mTOR signalling pathway and mitochondrial physiology were assessed using Western blot and flow cytometry.
\end{abstract}

Results: Intravenous HGF effectively alleviated pulmonary vascular endothelial injury and acute lung injury in the septic mice. The TEM results of lung tissue revealed that HGF attenuated pulmonary vascular endothelial pyroptosis, which was confirmed in vitro. Transfected LPS induced the pyroptosis of EA.hy926 cells and damaged their paracellular permeability, and these effects were ameliorated by treating the cells with recombinant HGF. The protective effect of HGF against pyroptosis was dependent on c-Met/mTOR signalling. mTOR activation effectively protected mitochondrial physiology and decreased reactive oxygen species (ROS) production in EA.hy926 cells in vitro.

Conclusions: These results demonstrated that HGF protected mitochondrial physiology by activating mTOR signalling to partially ameliorate endothelial pyroptosis and attenuate vascular endothelial injury and acute lung injury in sepsis animal model.

Keywords: Sepsis, Endothelial injury, Pyroptosis, HGF, mTOR, Mitochondria physiology

\footnotetext{
* Correspondence: yiyiyang2004@163.com

Jiangsu Provincial Key Laboratory of Critical Care Medicine, Department of Critical Care Medicine, Zhongda Hospital, School of Medicine, Southeast

University, 87 Dingjiaqiao Rd, Nanjing 210009, People's Republic of China
}

(c) The Author(s). 2020 Open Access This article is licensed under a Creative Commons Attribution 4.0 International License, which permits use, sharing, adaptation, distribution and reproduction in any medium or format, as long as you give appropriate credit to the original author(s) and the source, provide a link to the Creative Commons licence, and indicate if changes were made. The images or other third party material in this article are included in the article's Creative Commons licence, unless indicated otherwise in a credit line to the material. If material is not included in the article's Creative Commons licence and your intended use is not permitted by statutory regulation or exceeds the permitted use, you will need to obtain permission directly from the copyright holder. To view a copy of this licence, visit http://creativecommons.org/licenses/by/4.0/ The Creative Commons Public Domain Dedication waiver (http://creativecommons.org/publicdomain/zero/1.0/) applies to the data made available in this article, unless otherwise stated in a credit line to the data. 


\section{Introduction}

Sepsis is a common clinical syndrome defined as lifethreatening organ dysfunction caused by a dysregulated host response to infection [1]. Although numerous advances in management and treatment have been implemented to battle sepsis, the overall mortality rate remains as high as $35-45 \%$ [2, 3]. An in-depth exploration of novel therapeutic targets or strategies for sepsis is urgently needed.

One of the most important pathophysiological hallmarks of sepsis is vascular endothelial injury $[4,5]$, which rapidly results in tissue oedema and inflammation spread and is responsible for the progression of multiple organ failure during sepsis [5]. Although anticoagulant therapy and nitric oxide synthase inhibitors have shown protective effects in the endothelium, effective treatments for endothelial cell injury in sepsis that improve prognosis are lacking [6]. Therefore, it is of great significance and translational value to explore mechanisms and novel therapeutic options of endothelial cell injury.

Hepatocyte growth factor (HGF) is a pleiotropic cytokine involved in multiple cellular and biological processes, including improvement of cell injury and alleviation of inflammation. Previous studies demonstrated that lipopolysaccharide (LPS)-induced organ injury in rodents and that plasma HGF concentrations were increased in patients with systemic inflammatory response syndrome and early-phase sepsis [7, 8]; these increase of HGF are suggested to serve a compensatory mechanism to minimize LPS-induced cell and organ injury. Our previous studies found that mesenchymal stem cells (MSCs) with high HGF expression and secretion protect the pulmonary endothelial cell monolayer against LPS-induced hyperpermeability and monolayer integrity disruption and subsequently alleviate LPS intratracheal instillation-derived acute lung injury in rats [9, 10]. Although HGF was reported to have antiinflammatory effect on endothelial and protect the endothelial barrier in vitro, the molecular mechanisms underlying the protection against septic endothelial injury by HGF remain unclear.

Endothelial pyroptosis is a vital characteristic of septic endothelial injury. Pyroptosis is a recently recognized form of inflammatory programmed cell death that is different from apoptosis and necrosis in terms of molecular mechanisms and cellular representation; pyroptosis plays a critical role in the progression of sepsis [11]. Intracellular LPS or damage signalling activate inflammatory caspases and initiate pyroptosis, which is executed by gasdermin pores and ends with cell dissolution, with a massive release of damage-associated molecular patterns (DAMPs) [12]. Large amounts of endothelial pyroptosis destroying the endothelium were observed in the lungs and kidneys of septic mice [13, 14]. Although a small amount of evidence has revealed that HGF exerts antiapoptotic and cytoprotective effects in various epithelial and endothelial cells, e.g., HGF attenuates LPS-derived endothelial apoptosis [15], modulates chemotherapeutic agents induced autophagy and necrosis [16], whether HGF protects the vascular endothelium against pyroptosis in sepsis remains unknown.

This study investigated the role of HGF in the improvement of septic endothelial injury and the underlying mechanism. With the use of animal models, imaging studies, biochemical assays, and molecular inhibition approaches, we show that HGF ameliorates septic endothelial pyroptosis in vivo and in vitro and that the mammalian target of rapamycin (mTOR) signalling pathway plays a central role in this process.

\section{Materials and methods \\ Reagents}

Dulbecco's modified Eagle's medium (DMEM), foetal bovine serum (FBS), bovine serum albumin (BSA), recombinant HGF, 6-diamidino-2-phenylindole dihydrochloride (DAPI), and propidium iodide (PI) were obtained from Gibco (Grand Island, NY, USA). Lipopolysaccharide from Escherichia coli, serotype O127:B8, and penicillin/streptomycin were obtained from Sigma (St. Louis, MO, USA). The Lipofectamine 2000 reagent was purchased from Invitrogen (Carlsbad, CA, USA). Rapamycin and PHA-665752 were obtained from MCE (NJ, USA). The LDH cytotoxicity assay kits were from Beyotime Biotechnology (Nanjing, China). Interleukin (IL)-1 $\beta$, IL-18 and tumour necrosis factor (TNF)- $\alpha$ enzyme-linked immunosorbent assay (ELISA) kits were purchased from ExCellBio (Shanghai, China). The rabbit monoclonal phospho-Met antibody (Tyr1234/1235) (\#3077), rabbit monoclonal Akt (pan) antibody (C67E7) (\#4691), rabbit monoclonal phospho-Akt (Ser473) antibody (\#4050), rabbit monoclonal mammalian target of rapamycin (mTOR) antibody (\#2983S) were from Cell Signaling Technology (Beverly, MA, USA), and the rabbit monoclonal mTOR (phospho S2448) antibody (ab109268), rabbit monoclonal Met (c-Met) antibody (ab51067), and recombinant rabbit monoclonal pro Caspase- $1+$ p10 + p12 antibody (ab179515) were from Abcam (Cambridge, England, UK). The mouse monoclonal antibodies against gasdermin D (GSDMD) (sc-393, $581)$ and $\beta$-actin $($ sc-517,582) were obtained from Santa Cruz Biotechnology (Santa Cruz, CA, USA). The HRPconjugated IgG antibodies were from ZSGB-BIO (Beijing, China). All the other chemicals used in this study were of analytical grade and were obtained from Sigma (St. Louis, MO, USA) or Beyotime Biotechnology (Nanjing, China) unless otherwise stated. 


\section{Cell culture and treatment}

The human umbilical vein cell line (EA.hy926 cells) was cultured with DMEM containing $10 \%$ FBS at $37^{\circ} \mathrm{C}$ for $24 \mathrm{~h}$ in $5 \% \mathrm{CO}_{2}$ and $95 \%$ air. At the end of the incubation, the cells were incubated with serum-free medium for $1 \mathrm{~h}$, and the culture grew to $80 \%$ confluence prior to the initiation of the experimental treatments. The cells were cultured in 6-well plates with $2 \mathrm{~mL}$ of DMEM containing 10\% FBS and transfected with $2.5 \mu \mathrm{g} / \mathrm{mL}$ LPS using $2 \mu \mathrm{L} / \mathrm{mL}$ Lipofectamine 2000 (Lipo2000). Then, the stimulated cells were treated with $25 \mathrm{ng} / \mathrm{mL}$ HGF immediately and $6 \mathrm{~h}$ later, with or without $50 \mathrm{nM}$ PHA665752 or $20 \mathrm{nM}$ rapamycin.

\section{Mice}

C57BL/6 J (B6) mice were bred and maintained under specific pathogen-free conditions at Southeast University. Six- to 8-week-old mice were subjected to caecal ligation and puncture (CLP). Mortality was assessed every 3-4 h. In some sepsis treatment experiments, $1 \mu \mathrm{g} /$ g HGF was subsequently intravenously administered to the mice via the tail vein immediately and at $12 \mathrm{~h}$ after the CLP operation [17]. As a control, equal amounts of normal saline were administered in the same manner. To ensure that all regions were analysed with equal probability, an unbiased sampling cascade, systematic uniform random sampling (SURS), was applied, as recommended by the European Respiratory Society and the American Thoracic Society [18]. This study followed the national guidelines and protocols of the National Institutes of Health and was approved by the Local Ethics Committee for the Care and Use of Laboratory Animals of Southeast University.

\section{Evans blue pulmonary transvascular flux measurements}

We performed an Evans blue extravasation assay to measure vessel endothelial permeability. Briefly, Evans blue $(20 \mathrm{mg} / \mathrm{kg})$ in $1 \mathrm{~mL}$ saline was injected into the mice and allowed to circulate in the blood vessels for 1 h. Intravascular Evans blue was washed by heparinized normal saline perfusion from the right ventricle for 2 min. The mouse lungs were excised, weighed, homogenized in $1 \mathrm{~mL}$ PBS, and extracted overnight in $2 \mathrm{~mL}$ formamide at $60^{\circ} \mathrm{C}$. The Evans blue concentration in the lung homogenate supernatants was quantified by the spectrophotometric method at absorbances of 620 and $740 \mathrm{~nm}$. The lung weight/body weight $(\mathrm{LW} / \mathrm{BW})$ ratio was calculated to measure the pulmonary oedema.

\section{Lung histopathology}

For histopathological examination, the lung tissues were harvested, fixed in $4 \%$ paraformaldehyde for $24 \mathrm{~h}$, embedded in paraffin and cut into 4- $\mu$ m-thick sections, followed by H\&E staining. The sections were scanned by microscopy, and the lung injury score was quantified based on the images of 6 randomly chosen fields, following five criteria: oedema, alveolar and interstitial inflammation, alveolar and interstitial haemorrhage, atelectasis, and hyaline membrane formation. Each criterion was graded according to a 5-point scale (0-4) [19], more details about the scales are in the Additional file 1. The total lung injury score was calculated as the sum of the five criteria.

\section{TEM examination}

For transmission electron microscopy (TEM) examination, lung tissues and stimulated cells were harvested and cut into $<1-\mathrm{mm}^{3}$ sections and fixed in ice-cold $2.5 \%$ glutaraldehyde for $2 \mathrm{~h}$. Subsequently, the samples were postfixed in $1 \%$ osmium tetroxide for $1 \mathrm{~h}$, dehydrated through an ethanol series (50, 70, 95 and 100\%) and embedded in epoxy resin. Finally, the ultrathin sections $(60-80 \mathrm{~nm})$ were double-stained with uranyl acetate and lead citrate and examined via TEM (Hitachi HT7700, Tokyo, Japan). For the lung tissue sections, the vascular endothelial cells were scanned, and the ultrastructural details of the pyroptotic endothelial cells were analysed. For the cell sections, the cells were randomly selected to measure mitochondrial injury by a blind observer.

\section{Flow cytometry}

For the mitochondrial physiology assay, ROS levels and mitochondrial activity were measured by flow cytometry. EA.hy926 cells were treated as described, stained with DCFH-DA and MitoTracker for $30 \mathrm{~min}$, and then analysed with flow cytometry. Data were acquired from 50, 000 events using a BD LSR Fortessa (BD Biosciences), and the data were analysed by BD FACSDiva (BD Biosciences). The percentage of ROS-FLICA- or MitoTracker-APC-stained cells was analysed.

\section{Immunofluorescence}

EA.hy926 cells cultured on glass coverslips were treated as described above and washed with PBS. After staining with MitoTracker for $30 \mathrm{~min}$ and washing with PBS, the cells were stained with DAPI for nuclear counterstaining. The stained slides were photographed using a fluorescence inversion microscope system (Olympus, Tokyo, Japan), the cells were randomly selected to measure mitochondrial injury.

\section{Western blot}

EA.hy926 cells were cultured in 6-well plates and treated according to the experimental design described above. Then, whole cell lysates were harvested from the EA.hy926 cell monolayers. The total protein was quantified using the BCA method and adjusted to equal amounts. The protein mixtures were separated via $10 \%$ 
SDS-PAGE and transferred to active polyvinylidene difluoride membranes. After the transfer, the membranes were blocked with 5\% BSA-TBST for $1 \mathrm{~h}$ at room temperature and then probed with primary antibodies against caspase-1 (1:200), GSDMD (1:1000), Met (1: 1000), p-Met (1:1000), AKT (1:1000), p-AKT (1:1000), mTOR (1:1000) and p-mTOR (1:1000) at $4{ }^{\circ} \mathrm{C}$ overnight. The membranes were probed with an anti- $\beta$ actinantibody (1:1000) to control for protein loading and then incubated for $2 \mathrm{~h}$ at room temperature with HRPconjugated secondary antibodies (1:1000). The results were scanned using a gel imaging system (UVP Company, Upland, CA, USA). Densitometry measurements were performed with Image Lab software (Bio-Rad Laboratories, Hercules, CA, USA).

\section{$\mathrm{LDH}$ release}

EA.hy926 cells were cultured overnight in 96-well plates and transfected with $2.5 \mu \mathrm{g} / \mathrm{mL}$ LPS using Lipofectamine 2000 reagent for $12 \mathrm{~h}$. The culture medium was collected and analysed using an LDH cytotoxicity assay kit according to the manufacturer's instructions. The absorbance was measured at a wavelength of $490 \mathrm{~nm}$. The LDH release of each sample well was calculated by dividing the positive well after subtracting the negative well.

\section{Elisa}

EA.hy926 cells were cultured overnight in 24-well plates and transfected with $2.5 \mu \mathrm{g} / \mathrm{mL}$ LPS using Lipofectamine 2000 reagent for $12 \mathrm{~h}$. The culture medium was collected and analysed by IL-1 $\beta$ and IL-18 ELISA kits according to the manufacturer's instructions. The absorbance was measured at a wavelength of $450 \mathrm{~nm}$. The concentrations of the cytokines in each sample well were calculated based on a concurrent standard curve.

\section{Statistical analysis}

The data are expressed as the mean \pm standard deviation on the basis of at least three separate experiments. The data were analysed using SPSS version 23.0 (SPSS Inc., Chicago, IL, USA). Significant differences amongst the mean values of multiple groups were evaluated with one-way ANOVA followed by Student-Newman-Keuls' method. Survival was analysed using the log-rank test. A two-sided $P$ value $<0.05$ was considered statistically significant.

\section{Results}

\section{HGF effectively alleviated acute lung injury in sepsis}

To explore the effect of HGF on acute lung injury (ALI) in sepsis, we intravenously injected recombinant HGF into mice with sepsis caused by caecal ligation puncture (CLP) (Fig. 1a). Histopathological examination showed severe inflammatory cell infiltration, alveolar injury, interstitial oedema, and alveolar collapse in the lung tissues from the septic mice compared with those from the sham mice $12 \mathrm{~h}$ after the procedure (Fig. 1b). Compared to CLP alone, HGF treatment dramatically abated the inflammation and alleviated the lung injury induced by polymicrobial sepsis (Fig. 1c-d). With the increased invasion of a plethora of immunocytes, a surge in cytokines, such as IL-1 $\beta$, IL-18, and lactate dehydrogenase (LDH), was observed in the plasma and bronchoalveolar lavage fluid (BALF) from the septic mice in the CLP group; however, this effect was abrogated by intravenous administration of HGF in the CLP + HGF group (Fig. 1eh). In addition, the administration of recombinant HGF significantly reduced the mortality of the septic mice (Fig. 1i). There was no significant difference between the Sham and Sham+HGF groups.

\section{HGF attenuated pulmonary vascular endothelial injury in septic mice}

Endothelial injury was the primary contributor to interstitial oedema and alveolar injury in acute lung injury in sepsis. To investigate the effect of HGF on endothelial injury, we measured the pulmonary vascular permeability of the septic mice after the administration of recombinant HGF. As shown in the results of the Evans blue assay, the vascular permeability was higher in the septic mice than in the Sham mice, which was consistent with the protein levels in the BALF (Fig. 2a-b). Compared to CLP alone, intravenous administration of recombinant HGF dramatically decreased the effusion of Evans blue and protein, clearly indicating improved permeability (Fig. 2a-b). Severe pulmonary oedema was also detected in the septic mice, as revealed by lung weight/body weight (LW/BW) ratio, and this pulmonary oedema was abrogated by HGF (Fig. 2c) in the CLP + HGF group. There was no significant difference between the Sham and Sham+HGF groups.

Ultrathin lung sections were scanned by TEM to assess pulmonary vascular endothelial injury. A number of pyroptotic morphological features, such as cytoplasmic swelling, bubbling, osmotic lysis, nuclear condensation and oligonucleosomal DNA fragmentation, appeared in the pulmonary vascular endothelial cells in the ultrathin sections from the septic mice. However, endothelial pyroptosis was mitigated in the septic mice by the administration of HGF (Fig. 2d).

\section{HGF improved endothelial pyroptosis in vitro}

To confirm the effect of HGF on endothelial pyroptosis, umbilical vein endothelial pyroptosis was induced by transfected LPS (tLPS) in vitro. The EA.hy926 cells presented a typical pyroptotic morphology, including cell swelling, membrane rupture, bubbling, and bubble-like cell protrusions, and a large amount of LDH and IL-1 $\beta$ was released into the extracellular milieu after LPS 


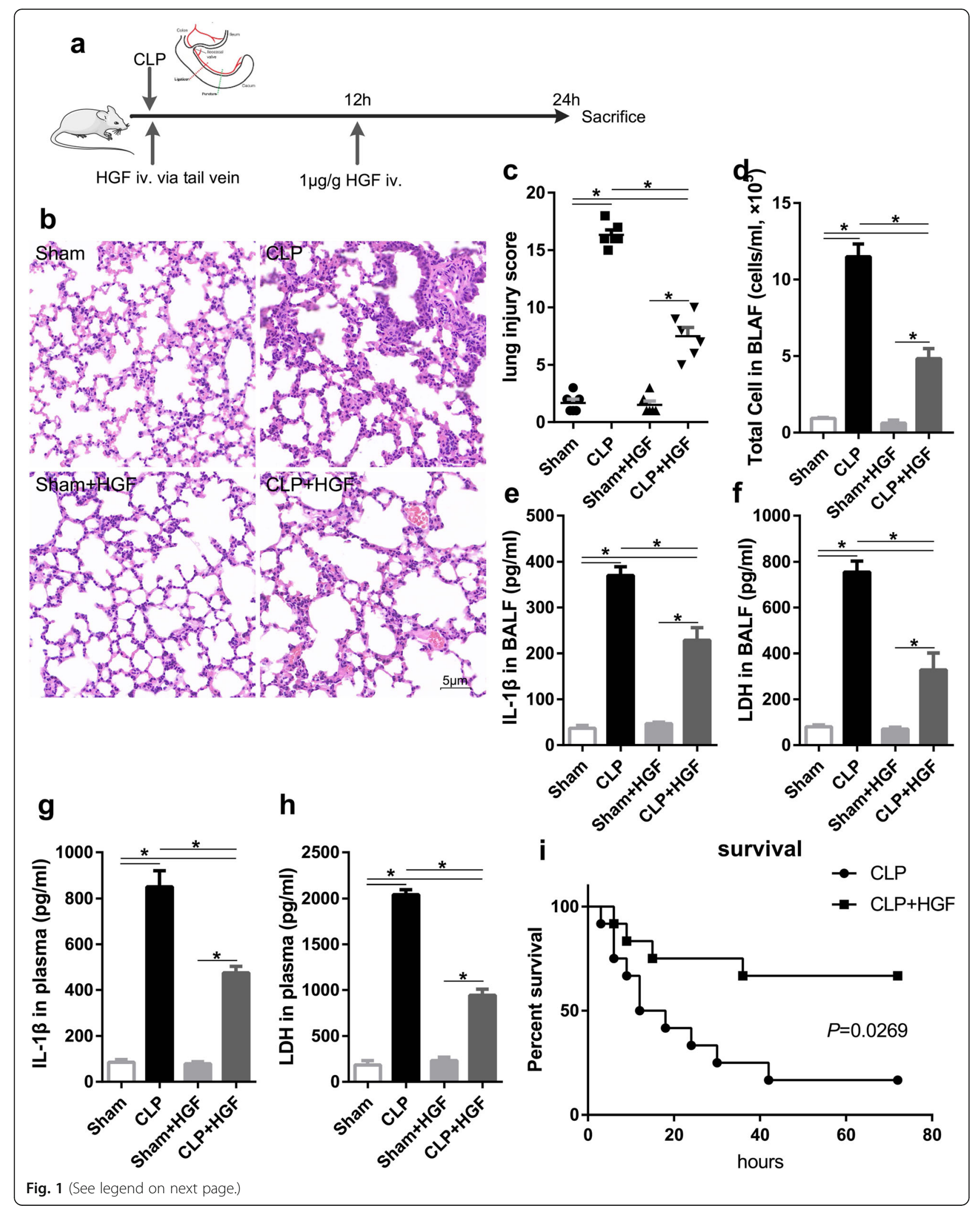


(See figure on previous page.)

Fig. $1 \mathrm{HGF}$ alleviated acute lung injury in sepsis. C57BL/6 J mice were randomly assigned to 4 groups. a The HGF treatment schedule, recombinant HGF $(1 \mathrm{\mu g} / \mathrm{g})$ was intravenously administered to the mice via the tail vein immediately and at $12 \mathrm{~h}$ after the operation. The mice were sacrificed $24 \mathrm{~h}$ after the operation. $\mathbf{b}$ Lung histopathological features; $\mathbf{c}$ Lung injury score, six random fields in a section from each mouse were photographed and assessed; $\mathbf{d}$ Total cell number in the BALF (cells $/ \mathrm{ml}, \times 10^{5}$ ) was counted by Cell Counter; $\mathbf{e}, \mathbf{f} \| \mathrm{L}-1 \beta$ and LDH in the BALF $(\mathrm{pg} / \mathrm{ml})$ were measured by ELISA; $\mathbf{g}, \mathbf{h} I \mathrm{~L}-1 \beta$ and LDH in the plasma $(\mathrm{pg} / \mathrm{ml})$ were measured by ELISA; $n=3,{ }^{*} P<0.05$; (I) Mortality was assessed every $3-4 h$, the survival was monitored until $72 \mathrm{~h}$, and the survival curve was analysed, $n=12$

transfection (Fig. 3a-d). LPS stimulation also promoted, to some extent, LDH release and IL-1 $\beta$ secretion by EA.hy926 cells (Fig. 3c-d) but did not induce pyroptosis (Fig. 3a). However, recombinant HGF significantly alleviated pyroptosis and decreased LDH and IL-1 $\beta$ release (Fig. 3a-d). Gasdermin D (GSDMD) and caspase-1 cleavage in endothelial cells were decreased by HGF administration (Fig. 3e). HGF dramatically protected mitochondrial integrity and decreased ROS production in pyroptotic endothelial cells (see Additional file 1 Fig. S1).

\section{HGF ameliorated endothelial pyroptosis by promoting mTOR signalling}

A crucial mechanism of growth factor-induced downstream biological effects is the constitutive activation of mTOR [20]. HGF specifically binds to the receptor cMet and then activates the key downstream AKT/mTOR signalling pathway to play a crucial role in cell survival and other programmed cell death, such as apoptosis and autophagy [21]. To test whether the c-Met/mTOR signalling pathway mediates the protective role of $\mathrm{HGF}$ against endothelial pyroptosis, PHA-665752 (PHA) and
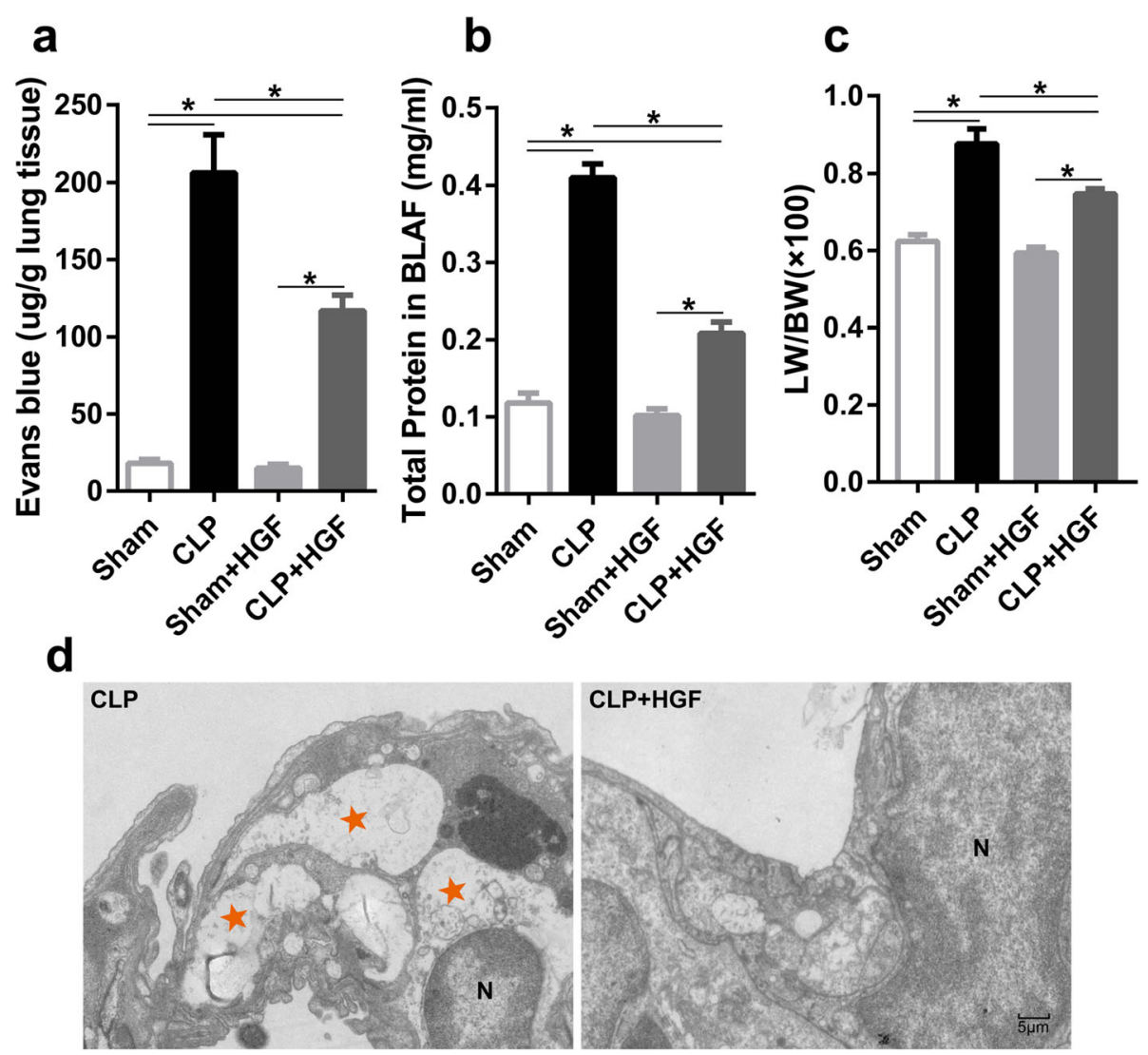

Fig. 2 HGF attenuated pulmonary vascular endothelial injury in septic mice. a Evans Blue was intravenously administered and measured in lung homogenates to assess pulmonary vascular permeability; $\mathbf{b}$ The total protein contents in the BALF were measured using the BCA method to assess protein leakage; $\mathbf{c}$ The lung weight and body weight ratio were analysed to assess lung oedema; $n=3,{ }^{*} P<0.05$; (D) Ultrathin lung sections were scanned using TEM to assess vascular endothelial injury by a blind observer; $N$ indicates nuclear; pentagram indicates bubbling of pyroptotic cells; scale bar $=5 \mu \mathrm{m}$ 

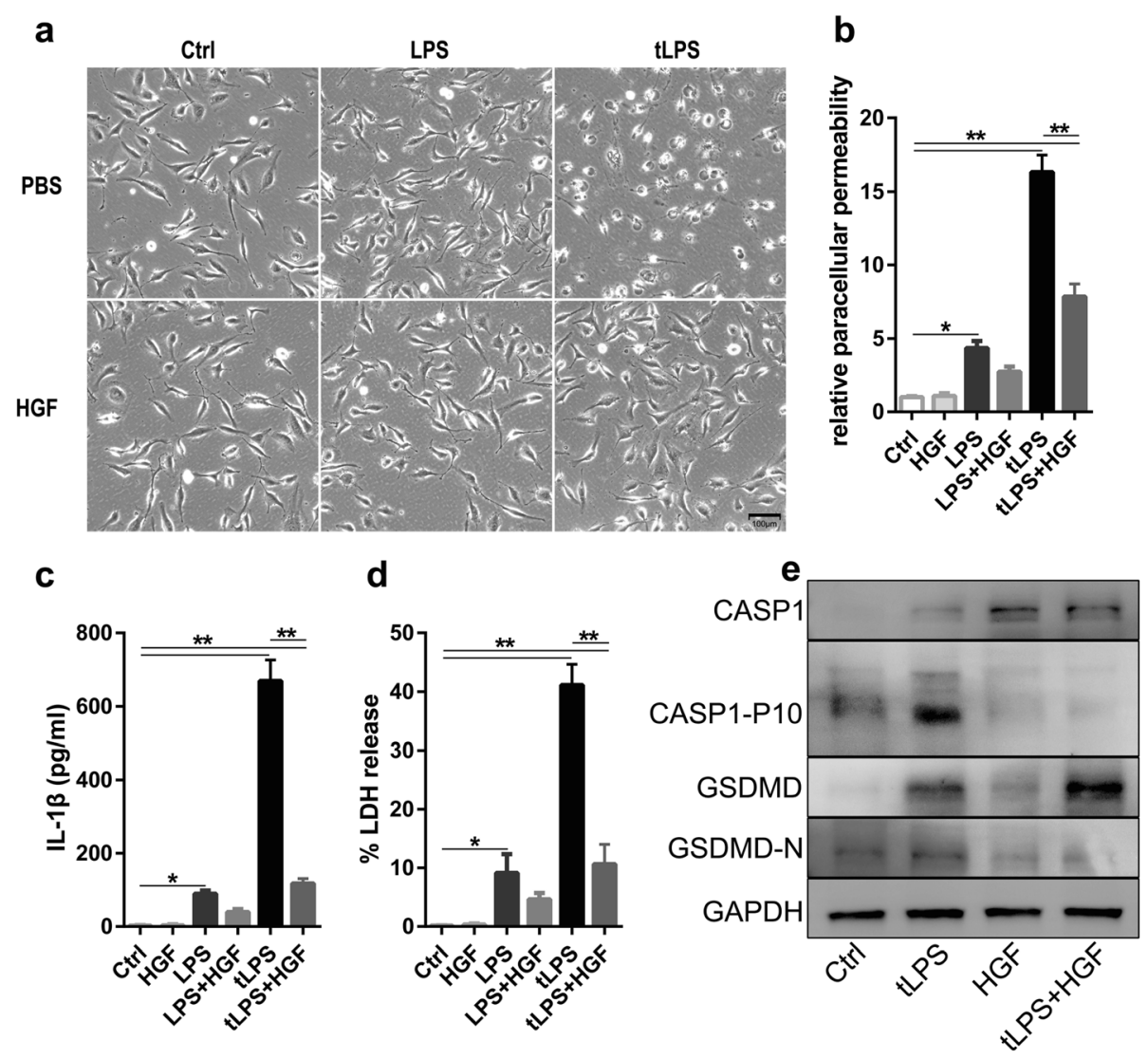

Fig. $3 \mathrm{HGF}$ alleviated endothelial pyroptosis in vitro. EA.hy926 cells were stimulated with LPS $(2.5 \mu \mathrm{g} / \mathrm{mL})$ with or without Lipo $2000(2 \mu \mathrm{L} / \mathrm{mL})$, followed by administration of HGF $(25 \mathrm{ng} / \mathrm{mL})$ immediately and $6 \mathrm{~h}$ later. a Bright-field image of treated EA.hy926 cells from randomly selected fields of view, in which morphological changes characteristic of pyroptosis were assessed; scale bar $=100 \mu \mathrm{m}$; b Relative paracellular permeability of single-layer EA.hy 926 cells exposed to different treatments and analysed by FITC-dextran; $\mathbf{c}$ IL-1 $\beta$ in the supernatant of treated EA.hy 926 cells was measured by ELISA; d LDH release assay was performed to measure pyroptosis of the treated EA.hy 926 cells, $n=3,{ }^{*} P<0.05$, ${ }^{*} P<0.01$; e CASP-1, CASP-1-P10, GSDMD and GSDMD-N in the treated EA.hy926 cell homogenates were measured by Western blot; $n=3$

rapamycin (RAPA), specific inhibitors of Met and mTOR, respectively, were administered to the endothelial cells. Immunoblotting revealed that c-Met in the plasma membrane was phosphorylated in response to the binding of HGF, and this c-Met phosphorylation was abolished by PHA-665752 in the LPS-transfected endothelial cells (Fig. 4). In addition, HGF treatment dramatically phosphorylated mTOR-Ser2448 and AKT-Ser473, which indicate the activation of mTOR [22], in pyroptotic endothelial cells. However, mTOR activation was abrogated by rapamycin, a specific inhibitor of mTOR, as evidenced by the weak expression of P-AKT-Ser473 and P-mTOR-Ser2448 (Fig. 4).

HGF administration remarkably improved the endothelial cell pyroptosis caused by LPS transfection, as revealed by the morphological features observed by bright field microscopy, and this effect was obviously abolished by the inhibitor of c-Met, PHA-665752 (PHA, $50 \mathrm{nM}$ ), and the inhibitor of mTOR, and rapamycin (RAPA, 20 $\mathrm{nM}$ ) (Fig. 5a). The inhibitory effect of HGF on IL-1 $\beta$ secretion and LDH release by pyroptotic endothelial cells was impaired by PHA-665752 and rapamycin (Fig. $5 c, d)$. Moreover, the inhibition of GSDMD or caspase-1 cleavage by HGF was abolished by PHA-665752 and rapamycin (Fig. 5e). HGF alleviated endothelial cell pyroptosis, and this effect was dependent on the promotion of mTOR signalling.

\section{HGF protected mitochondrial physiology by promoting mTOR signalling}

Although mTOR signalling was confirmed to govern mitochondrial integrity and function in previous studies [23], whether HGF/c-Met considerably directs crosstalk remains poorly defined. We assessed mitochondrial integrity and function after HGF administration in LPStransfected EA.hy.926 cells. As the flow cytometry and immunofluorescence results demonstrated, HGF dramatically protected mitochondrial integrity and decreased ROS production in the pyroptotic endothelial cells (Fig. 6a-c). Mitochondrial injury in the tLPS-treated 


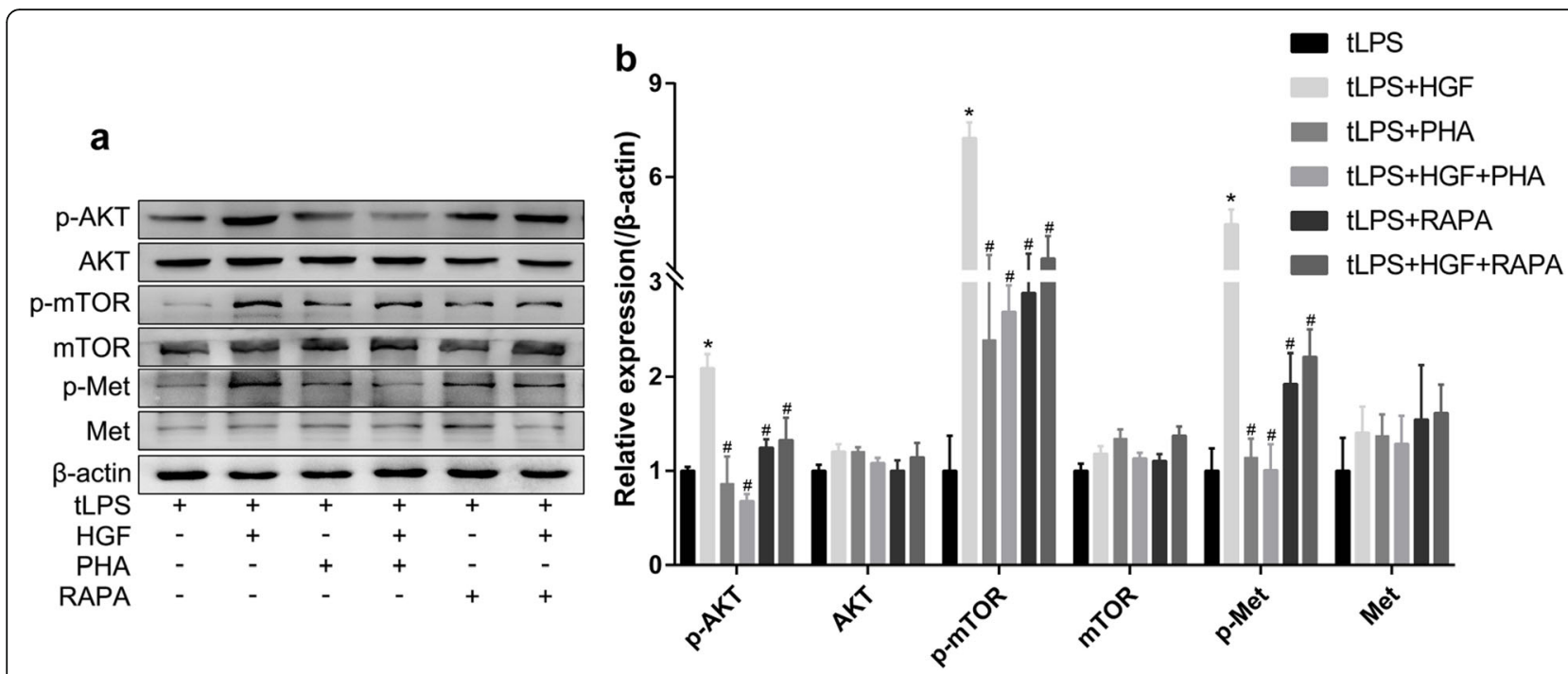

Fig. 4 c-Met/mTOR signalling pathway was measured by Western blot. EA.hy926 cells were stimulated with Lipo $2000(2 \mu \mathrm{L} / \mathrm{mL}) \mathrm{and} \mathrm{LPS}(2.5 \mu \mathrm{g} /$ $\mathrm{mL})$, followed by administration of HGF $(25 \mathrm{ng} / \mathrm{mL})$ with or without PHA-665752 $(50 \mathrm{nM})$ or rapamycin $(20 \mathrm{nM})$ immediately and $6 \mathrm{~h}$ later. a Met, p-Met-Tyr1234/1235, mTOR, p-mTOR-Ser2448, AKT and p-AKT-Ser473 in the treated EA.hy926 cell homogenates were measured by Western blot; b Quantification of relative expression, $n=3$; ${ }^{*} P<0.05$ compared with the tLPS group; \#P< 0.05 compared with the tLPS+HGF group; PHA: PHA665752, RAPA: rapamycin.

EA.hy926 cells was mitigated by HGF, which was also evidenced by the TEM results (Fig. 6d). However, all the protective effects of HGF were abrogated by the inhibition of mTOR.

\section{Discussion}

The results of our study demonstrated that HGF effectively ameliorates sepsis-induced acute lung injury and pulmonary vascular endothelial injury. The protective role of HGF involves inhibiting endothelial pyroptosis by protecting mitochondrial physiology through the mTOR signalling pathway. To the best of our knowledge, this is the first report that describes the mechanism by which the mTOR signalling pathway plays a key role in the amelioration of endothelial pyroptosis by HGF.

HGF is a multifunctional growth factor that is involved in a variety of physiological activities and pathological processes. Enhancement of endogenous HGF response for minimizing septic tissue injuries, however, secretion of HGF seems insufficient in sepsis, with a time lag between the injury and HGF increasing. Here, we demonstrated that HGF alleviates polymicrobial sepsis-induced lung injury and inflammation and improves the survival rate of septic mice. Previous studies have illustrated that HGF alleviates acute kidney injury and acute hepatic injury in mice with LPS-induced sepsis $[24,25]$. Several lines of clinical evidence have demonstrated that blood HGF levels raised in response to organ damage in patients with sepsis in the early phase [8]. Therefore, this experimental result indicates that HGF supplement therapy might be available for improving sepsis-induced organ damage. Endothelial injury and lung injury were not fully reversed by HGF, probably due to the complex pathophysiological mechanism of polymicrobial sepsis and the subsequent injury induced by persistent inflammation, nor did recombinant HGF rescue all of septic mice in previous researches [26]. An efficient dose of HGF $(1 \mu \mathrm{g} / \mathrm{g})$ was chosen to intravenously treat septic mice immediately and at $12 \mathrm{~h}$ after CLP operation, because the lung is the most susceptible organ. Kamimoto $\mathrm{M}$ et al. [27] reported HGF treatment was gaven twice 1 day after LPS challenge 0 and $12 \mathrm{~h}$. Kosai $\mathrm{K}$ et al. [26] gave recombinant HGF intraperitoneally injections $6 \mathrm{~h}$ before and $3 \mathrm{~h}$ after LPS injection, because only the few hours from liver injury to death. However, for clinical treatment of sepsis with HGF, clinical studies are necessary, the dose and timing of HGF administration should be determined according to its pharmacokinetics and pharmacodynamics in patients. Therefore, HGF may be a promising supplemental therapy to ameliorate sepsisinduced organ damage.

Endothelial pyroptosis is a vital mechanism of vascular endothelial injury in sepsis, and this process leads to the release of a plethora of pro-inflammatory cytokines, such as IL- $1 \beta$ and LDH, and destroys the endothelium barrier, eventually leading to septic shock and multiple organ failure $[13,14,28]$. The specific inhibition of endothelial pyroptosis attenuates LPS-induced ALI and decreases sepsis-induced mortality in Casp $11^{E C-/-}$ mice [9], which means that endothelial pyroptosis is a promising therapeutic target. The present study illustrated that HGF effectively inhibited endothelial pyroptosis, reduced 


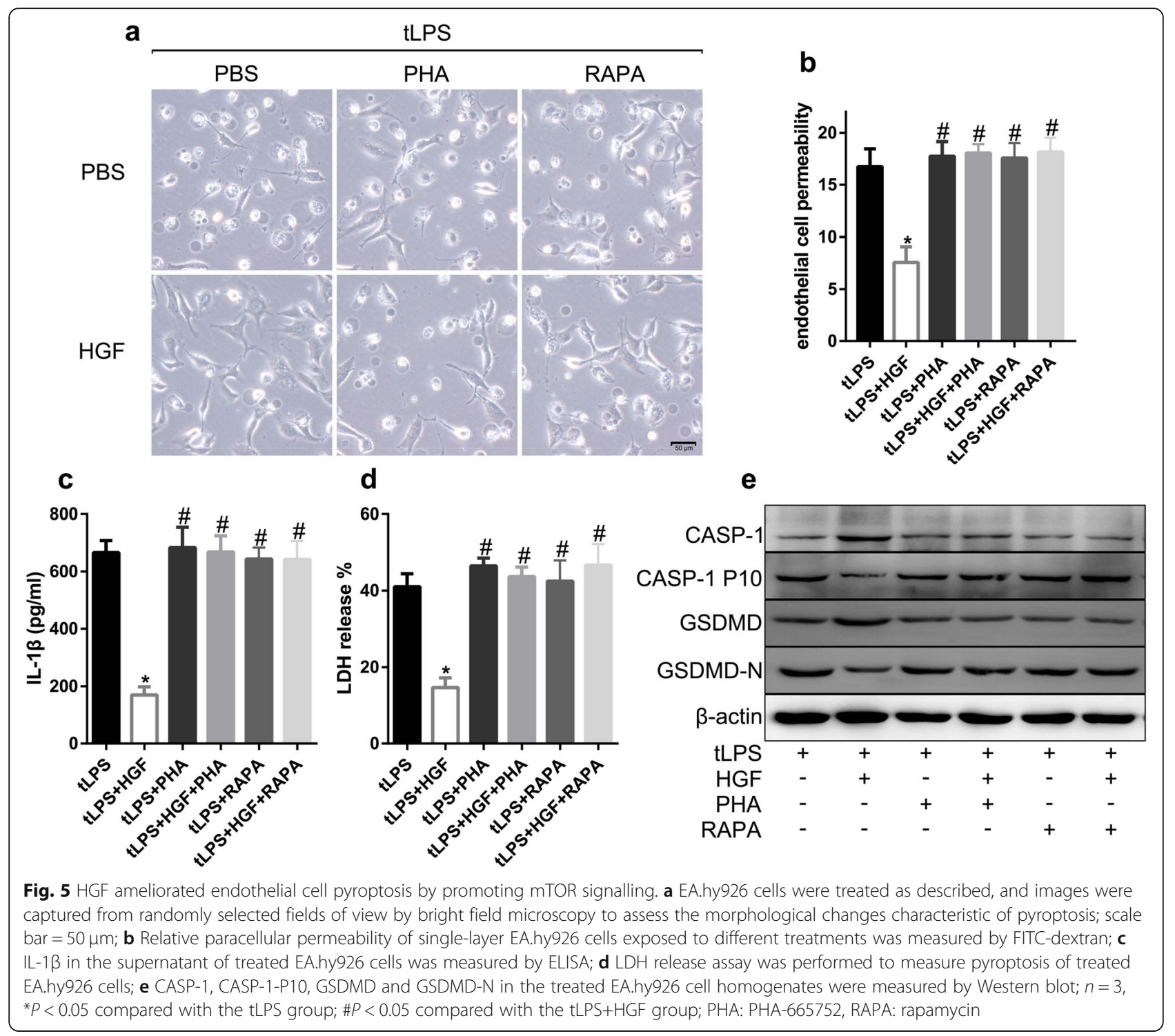

vascular permeability, and decreased IL- $1 \beta$ and LDH secretion. Although previous studies have shown that HGF has anti-apoptotic and anti-necrotic effects, this is the first study reporting its anti-pyroptotic effect, which is meaningful for dissecting the mechanism by which HGF repairs endothelial injury.

Mitochondrial damage is a crucial contributor to and hallmark of pyroptosis [29, 30]. Many stress factors, such as microbiome metabolites, toxicants and oxidized microenvironments, have been shown to disrupt mitochondrial homeostasis [31]. In addition, the gasdermin pore in the plasma membrane eventually executes pyroptosis, simultaneously causing the mitochondria to release its contents [32]. The ROS, mtDNA, and ATP released from injured mitochondria strongly promote pyroptosis by activating the inflammasome and the cleavage of caspase-1 [33-35]. Our results have shown that HGF protects the integrity of the mitochondrial plasma membrane, reduces the release of mitochondrial contents, leads to the scavenging of ROS or other mitochondrial damage-associated molecules and prevents pyroptosis $[36,37]$. Thus, improving mitochondrial physiology alleviates endothelial pyroptosis and may be a therapeutic target for sepsis.

HGF binds to c-Met in the plasma membrane, activates the $A K T / m T O R$ signalling pathway, plays a vital role in cell growth, metabolism, cell survival and migration; in addition, HGF is closely associated with developmental defects, cancer, diabetes and autoimmune diseases [22, 38]. Our results demonstrated that HGF activates the AKT/mTOR signalling pathway to protect mitochondrial physiology and reduce pyroptosis in endothelial cells. Previous studies have proven that mTOR controls the structure and function of 


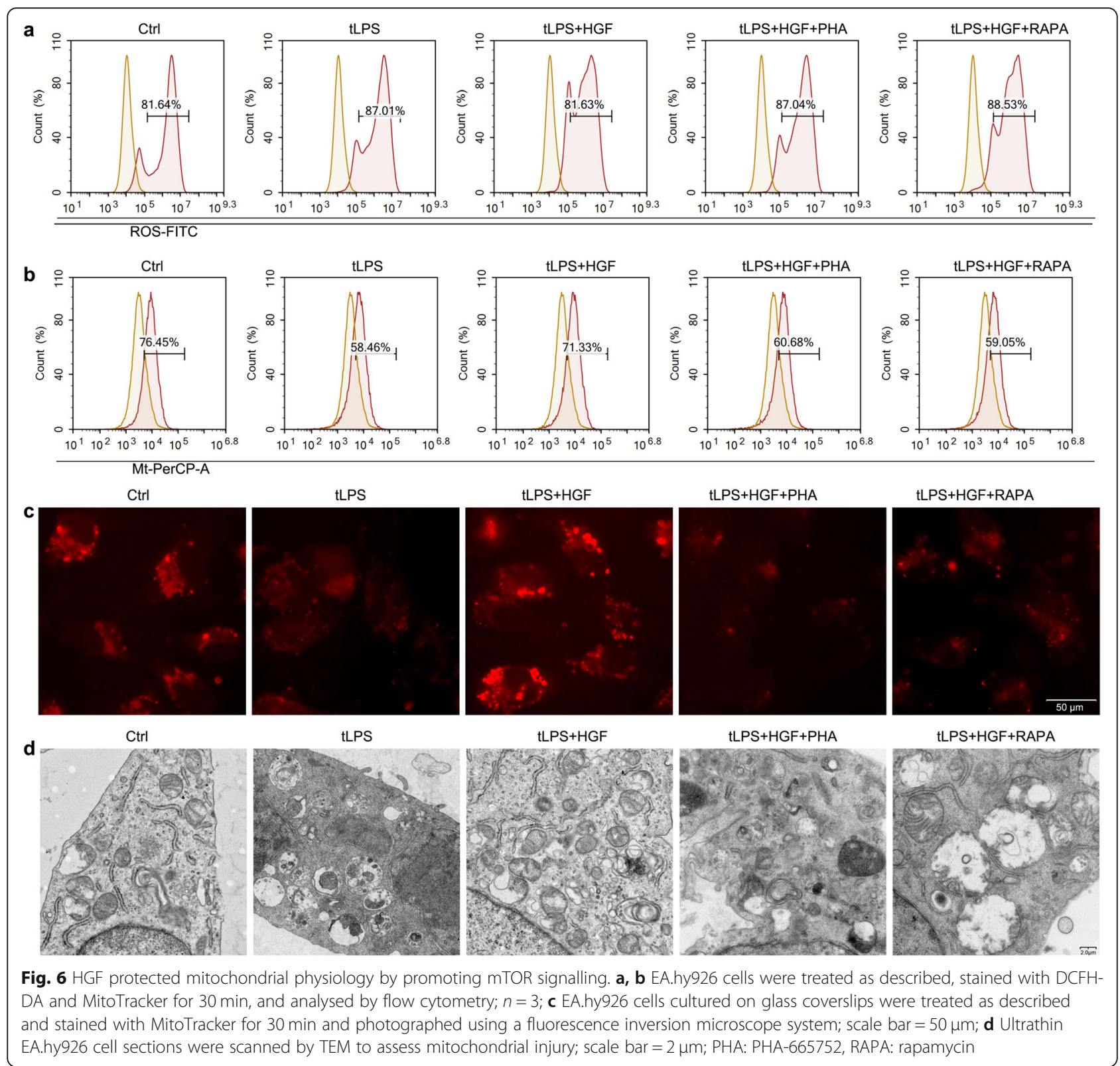

mitochondria. mTOR complex 1 selectively promotes the translation of nucleus-encoded, mitochondriarelated mRNAs to control mitochondrial activity and biogenesis [39]. mTOR complex 2 localizes to the plasma membrane of mitochondria to mediate its integrity and control mitochondrial physiology [23]. Thus, mTOR signalling appears to be a particularly important hub for HGF in the repair of endothelial injury. Other downstream pathways of $\mathrm{HGF} / \mathrm{c}-\mathrm{Met}$, such as the MAPK, Ras/MEK, STAT3, ІкB $\alpha / \mathrm{NF}-\kappa B$ pathways, were reported to mediate invasive growth, resist apoptotic insults and cause proliferattion. Although our previous study [15] revealed that following HGF stimulation, STAT3 was activated and endothelial apoptosis partially attenuated, we did not measure the effects of these pathways on endothelial pyroptosis here, which is a limitation.

In summary, we demonstrated that mTOR signalling mediates the protective effect of HGF on mitochondrial physiology. HGF inhibits the release of mitochondrial contents to alleviate endothelial pyroptosis in vitro. The effect of HGF in attenuating vascular endothelial injury could alleviate acute lung injury in sepsis and improve the prognosis of sepsis, at least in animal model. Although the effect of HGF on clinical sepsis patients is speculative and remains to be elucidated, it is tempting to believe that it could be a promising adjuvant therapy for sepsis. 


\section{Conclusion}

HGF ameliorates endothelial pyroptosis depending activation of mTOR signalling by protecting mitochondrial physiology, avoiding mitochondrial damage-associated molecular release in vitro. The recombinant HGF intravenously administration mitigates polymicrobial sepsis-induced pulmonary vascular endothelial pyroptosis, attenuates pulmonary vascular endothelial injury and acute lung injury in mice. HGF may have the potential to be a promising adjuvant therapeutic strategy aimed at the treatment of sepsis and acute lung injury.

\section{Supplementary information}

Supplementary information accompanies this paper at https://doi.org/10. 1186/s12931-020-01480-3

Additional file 1: Figure S1. HGF alleviated endothelial pyroptosis in vitro. EAhy926EA.hy926 cells were stimulated with LPS $(2.5 \mathrm{ug} / \mathrm{mL})$, lipo2000 (2uL/mL) and LPS $(2.5 \mathrm{ug} / \mathrm{mL})$ for $6 \mathrm{~h}$, followed by HGF administration $(25 \mathrm{ng} / \mathrm{mL}) 6 \mathrm{~h}$, respectively. EA.hy926 cells were treated as mentioned and stained with DCFH-DA and MitoTracker for 30min, measured by flow cytometry; $n=3$.

\section{Abbreviations}

Term: Definition; HGF: Hepatocyte growth factor; TEM: Transmission electron microscopy; LPS: Lipopolysaccharide; ROS: Reactive oxygen species;

DAMP: Damage-associated molecular patterns; MSC: Mesenchymal stem cell; CLP: Caecal ligation puncture; LDH: Lactate dehydrogenase;

BALF: Bronchoalveolar lavage fluid; ALI: Acute lung injury; LW/BW: Lung weight/body weight; tLPS: Transfected LPS; GSDMD: Gasdermin D;

BCA: Bicinchoninic acid method; RAPA: Rapamycin; mTOR: Mammalian target of rapamycin

\section{Acknowledgements}

No acknowledgements.

\section{Authors' contributions}

FP and YY designed the study; FP conducted the study; FP and WC analysed the data; FP, QS, XX, JX, and YY were involved in data interpretation; FP, WC, $\mathrm{HQ}$ and $Y Y$ wrote and revised the paper. All the authors read and approved the final manuscript.

\section{Funding}

This work is partially supported by grants from National Science and Technology Major Project for Control and Prevention of Major Infectious Diseases of China (Grant No. 2017ZX10103004), National Natural Science Foundation of China (Grant No. 81671892, 81971888), Jiangsu Province's Key Discipline/Laboratory of Medicine (Grant No. ZDXKA2016025), Jiangsu Province's Key Provincial Talents Program (Grant No. ZDRCA2016082), Natural Science Foundation of Jiangsu Province (Grant No. BK20161433) and Postgraduate Research \& Practice Innovation Program of Jiangsu Province (Grant No. KYCX18_0181).

\section{Availability of data and materials}

Figure 1S HGF protected mitochondrial physiology in vitro. EA.hy926 cells were treated as described, stained with DCFH-DA and MitoTracker for 30 min, analysed by flow cytometry; $n=3$.

\section{Ethics approval and consent to participate}

This study followed the national guidelines and protocols of the National Institutes of Health and was approved by the Local Ethics Committee for the Care and Use of Laboratory Animals of Southeast University.

\section{Consent for publication}

All authors provided consent for publication.

\section{Competing interests}

The authors declare that they have no competing interests.

Received: 1 June 2020 Accepted: 3 August 2020

Published online: 14 August 2020

\section{References}

1. Singer M, Deutschman CS, Seymour CW, Shankar-Hari M, Annane D, Bauer $M$, et al. The third international consensus definitions for Sepsis and septic shock (Sepsis-3). Jama. 2016;315:801-10.

2. Xie J, Wang H, Kang Y, Zhou L, Liu Z, Qin B, et al. The epidemiology of Sepsis in Chinese ICUs: a National Cross-Sectional Survey. Crit Care Med. 2020:48:e209-e218.

3. Weng L, Zeng XY, Yin P, Wang LJ, Wang CY, Jiang W, et al. Sepsisrelated mortality in China: a descriptive analysis. Intensive Care Med. 2018;44:1071-80.

4. Lee WL, Slutsky AS. Sepsis and endothelial permeability. N Engl J Med. 2010; 363:689-91.

5. Lelubre C, Vincent $J$. Mechanisms and treatment of organ failure in sepsis. Nat Rev Nephrol. 2018;14:417-27.

6. Aird WC. The role of the endothelium in severe sepsis and multiple organ dysfunction syndrome. Blood. 2003;101:3765-77.

7. Sakon M, Kita Y, Yoshida T, Umeshita K, Gotoh M, Kanai T, et al. Plasma hepatocyte growth factor levels are increased in systemic inflammatory response syndrome. Surg Today. 1996;26:236-41.

8. Sekine K, Fujishima S, Aikawa N. Plasma hepatocyte growth factor is increased in early-phase sepsis. J Infect Chemother. 2004;10:110-4.

9. Hu S, Li J, Xu X, Liu A, He H, Xu J, et al. The hepatocyte growth factorexpressing character is required for mesenchymal stem cells to protect the lung injured by lipopolysaccharide in vivo. Stem Cell Res Ther. 2016;7:66.

10. Yang Y, Chen QH, Liu AR, Xu XP, Han JB, Qiu HB. Synergism of MSCsecreted HGF and VEGF in stabilising endothelial barrier function upon lipopolysaccharide stimulation via the Rac1 pathway. Stem Cell Res Ther. 2015;6:250.

11. Xue Y, Enosi Tuipulotu D, Tan WH, Kay C, Man SM. Emerging activators and regulators of Inflammasomes and Pyroptosis. Trends Immunol. 2019;40: 1035-52.

12. Pfalzgraff A, Weindl G. Intracellular lipopolysaccharide sensing as a potential therapeutic target for Sepsis. Trends Pharmacol Sci. 2019;40:187-97.

13. Cheng KT, Xiong S, Ye Z, Hong Z, Di A, Tsang KM, et al. Caspase-11mediated endothelial pyroptosis underlies endotoxemia-induced lung injury. J Clin Invest. 2017;127:4124-35.

14. Chen Q, Yang Y, Hou J, Shu Q, Yin Y, Fu W, et al. Increased gene copy number of DEFA1/DEFA3 worsens sepsis by inducing endothelial pyroptosis. Proc Natl Acad Sci U S A. 2019;116:3161-70.

15. Meng SS, Guo FM, Zhang XW, Chang W, Peng F, Qiu HB, et al. mTOR/STAT3 pathway mediates mesenchymal stem cell-secreted hepatocyte growth factor protective effects against lipopolysaccharide-induced vascular endothelial barrier dysfunction and apoptosis. J Cell Biochem. 2019;120: 3637-50.

16. Huang X, Gan G, Wang X, Xu T, Xie W. The HGF-MET axis coordinates liver cancer metabolism and autophagy for chemotherapeutic resistance. Autophagy. 2019;15:1258-79.

17. Kamimoto M, Mizuno S, Matsumoto K, Nakamura T. Hepatocyte growth factor prevents multiple organ injuries in endotoxemic mice through a heme oxygenase-1-dependent mechanism. Biochem Biophys Res Commun. 2009:380:333-7

18. Hsia CC, Hyde DM, Ochs M, Weibel ER. An official research policy statement of the American Thoracic Society/European Respiratory Society: standards for quantitative assessment of lung structure. Am J Respir Crit Care Med. 2010;181:394-418.

19. Smith KM, Mrozek JD, Simonton SC, Bing DR, Meyers PA, Connett JE, et al. Prolonged partial liquid ventilation using conventional and high-frequency ventilatory techniques: gas exchange and lung pathology in an animal model of respiratory distress syndrome. Crit Care Med. 1997;25:1888-97.

20. Ebner M, Sinkovics B, Szczygiel M, Ribeiro DW, Yudushkin I. Localization of mTORC2 activity inside cells. J Cell Biol. 2017;216:343-53.

21. Liu GY, Sabatini DM. mTOR at the nexus of nutrition, growth, ageing and disease. Nat Rev Mol Cell Biol. 2020;21:183-203.

22. Saxton RA, Sabatini DM mTOR signaling in growth, metabolism, and disease. Cell. 2017;168:960-76. 
23. Betz C, Stracka D, Prescianotto-Baschong C, Frieden M, Demaurex N, Hall MN. Feature article: mTOR complex 2-Akt signaling at mitochondriaassociated endoplasmic reticulum membranes (MAM) regulates mitochondrial physiology. Proc Natl Acad Sci U S A. 2013;110:12526-34.

24. Mizuno S, Nakamura T. Improvement of sepsis by hepatocyte growth factor, an anti-inflammatory regulator: emerging insights and therapeutic potential. Gastroenterol Res Pract. 2012;2012:909350.

25. Meng F, Meliton A, Moldobaeva N, Mutlu G, Kawasaki Y, Akiyama T, et al. Asef mediates HGF protective effects against LPS-induced lung injury and endothelial barrier dysfunction. Am J Phys Lung Cell Mol Phys. 2015;308: L452-63.

26. Kosai K, Matsumoto K, Funakoshi H, Nakamura T. Hepatocyte growth factor prevents endotoxin-induced lethal hepatic failure in mice. Hepatology (Baltimore, Md). 1999;30:151-9.

27. Kamimoto M, Mizuno S, Nakamura T. Reciprocal regulation of IL-6 and IL-10 balance by HGF via recruitment of heme oxygenase- 1 in macrophages for attenuation of liver injury in a mouse model of endotoxemia. Int J Mol Med. 2009;24:161-70

28. Yang J, Zhao Y, Zhang P, Li Y, Yang Y, Yang Y, et al. Hemorrhagic shock primes for lung vascular endothelial cell pyroptosis: role in pulmonary inflammation following LPS. Cell Death Dis. 2016;7:e2363.

29. de Vasconcelos NM, Van Opdenbosch N, Van Gorp H, Parthoens E, Lamkanfi M. Single-cell analysis of pyroptosis dynamics reveals conserved GSDMDmediated subcellular events that precede plasma membrane rupture. Cell Death Differ. 2019;26:146-61.

30. Yu J, Nagasu H, Murakami T, Hoang H, Broderick L, Hoffman HM, et al. Inflammasome activation leads to Caspase-1-dependent mitochondrial damage and block of mitophagy. Proc Natl Acad Sci U S A. 2014;111:15514-9.

31. Mills EL, Kelly B, O'Neill LAJ. Mitochondria are the powerhouses of immunity. Nat Immunol. 2017;18:488-98.

32. Rogers $C$, Erkes DA, Nardone A, Aplin AE, Fernandes-Alnemri T, Alnemri ES, Gasdermin pores permeabilize mitochondria to augment caspase-3 activation during apoptosis and inflammasome activation. Nat Commun. 2019;10:1689.

33. Zhong Z, Liang S, Sanchez-Lopez E, He F, Shalapour S, Lin XJ, et al. New mitochondrial DNA synthesis enables NLRP3 inflammasome activation. Nature. 2018;560:198-203.

34. Prochnicki T, Latz E. Inflammasomes on the crossroads of innate immune recognition and metabolic control. Cell Metab. 2017;26:71-93.

35. Li S, Hu Q, Huang J, Wu X, Ren J. Mitochondria-derived damage-associated molecular patterns in Sepsis: from bench to bedside. Oxidative Med Cell Longev. 2019;2019:6914849.

36. Martinez-Garcia JJ, Martinez-Banaclocha H, Angosto-Bazarra D, de TorreMinguela C, Baroja-Mazo A, Alarcon-Vila C, et al. P2X7 receptor induces mitochondrial failure in monocytes and compromises NLRP3 inflammasome activation during sepsis. Nat Commun. 2019;10:2711.

37. Wu X, Zhang H, Qi W, Zhang Y, Li J, Li Z, et al. Nicotine promotes atherosclerosis via ROS-NLRP3-mediated endothelial cell pyroptosis. Cell Death Dis. 2018;9:171.

38. Imanishi J, Kamiyama K, Iguchi I, Kita M, Sotozono C, Kinoshita S. Growth factors: importance in wound healing and maintenance of transparency of the cornea. Prog Retin Eye Res. 2000;19:113-29.

39. Morita M, Gravel SP, Chenard V, Sikstrom K, Zheng L, Alain T, et al. mTORC1 controls mitochondrial activity and biogenesis through $4 \mathrm{E}-\mathrm{BP}$-dependent translational regulation. Cell Metab. 2013;18:698-711.

\section{Publisher's Note}

Springer Nature remains neutral with regard to jurisdictional claims in published maps and institutional affiliations.

Ready to submit your research? Choose BMC and benefit from:

- fast, convenient online submission

- thorough peer review by experienced researchers in your field

- rapid publication on acceptance

- support for research data, including large and complex data types

- gold Open Access which fosters wider collaboration and increased citations

- maximum visibility for your research: over $100 \mathrm{M}$ website views per year

At BMC, research is always in progress.

Learn more biomedcentral.com/submissions 